\title{
Field theories and exact stochastic equations for interacting particle systems
}

\author{
Alexei Andreanov ${ }^{1}$, Giulio Biroli ${ }^{1}$, Jean-Philippe Bouchaud ${ }^{2,3}$, Alexandre Lefèvre $^{1}$ \\ 1 Service de Physique Théorique, Orme des Merisiers - CEA Saclay, 91191 Gif sur Yvette Cedex, France. \\ 2 Service de Physique de l'État Condensé, Orme des Merisiers - CEA Saclay, 91191 Gif sur Yvette Cedex, France. and \\ 3 Science \& Finance, Capital Fund Management, 6 Bd Haussmann, 75009 Paris, France.
}

\begin{abstract}
We present a new approach to the dynamics of interacting particles with reaction and diffusion. Starting from the underlying discrete stochastic jump process we derive a general field theory describing the dynamics of the density field, which we relate to an exact stochastic equation on the density field. We show how our field theory maps onto the original Doi-Peliti formalism, allowing us to clarify further the issue of the 'imaginary' Langevin noise that appears in the context of reaction/diffusion processes. Our procedure applies to a wide class of problems and is related to large deviation functional techniques developed recently to describe fluctuations of non-equilibrium systems in the hydrodynamic limit.
\end{abstract}

Many problems of current interest in statistical physics involve strongly interacting particles that exhibit non trivial collective phenomena. One example is that of supercooled liquids, where the dynamics slows down dramatically as the glass transition is approached, due to the increasingly collective nature of the dynamics [1]. Other examples are given by systems of diffusing particles that branch and/or annihilate and that are subjected to an external drive; depending on the relative strength of these effects a variety of non-equilibrium transitions and anomalous scaling behaviour can appear [2]. Developing theoretical techniques for such difficult problems is of great importance, in view of the diversity of situations in which they can apply, and of much broader scope than usual equilibrium statistical mechanics. A natural framework in this context is that of field theory which combined with perturbative renormalization group techniques have been applied successfully to a wide range of non-equilibrium phase transitions 3]. Furthermore, even in non perturbative regimes, field theory can be very useful because it allows one to articulate different types of approximations, such as the Exact Renormalisation Group approach [4] and Mode Coupling Theory [5, 6]. A field theoretical formulation of interacting particle systems which has become somewhat standard in recent years is provided by the Doi-Peliti formalism (DP) 3]. Starting from a second quantization representation of the Master equation, one obtains, after a rather elaborate coherent state representation, a field theory in terms of two fields $\phi$ and $\hat{\phi}$ (see below) which has been the starting point of a very large number of studies [3]. However, besides its intrinsic complexity, the formalism appears to be fraught with difficulties. For example, the action of the field theory corresponds to a reasonable looking Langevin equation for the density of particles, except that the noise is often complex or even pure imaginary. This suggests that the field $\phi$, despite its superficial resemblance with the density, in fact lacks direct physical interpretation, see e.g. [7].

The aim of this Letter is to formulate a two-field theory for interacting particles in a transparent way, starting from a natural representation of the microscopic stochastic dynamics of the system in discrete space. Our formalism focuses on the physical density field $\rho$ and, as a consequence, is related directly to the stochastic equations governing the evolution of $\rho$. The original DP formalism is recovered performing a canonical Cole-Hopf transformation. Our method is straightforward, free of the ambiguities related to the imaginary noise and applies to many different situations (diffusion in an external force field, pair-wise interacting particles, branching/annihilation processes, hard-core particles etc.). Furthermore it is related to recent techniques developed in mathematical physics (see below) 8]. As an interesting physical side product, our field theory in the case of liquids is found to be identical to the one obtained from a Martin-Siggia-Rose-De Dominicis-Janssen (MSRDJ) 9] representation of the stochastic equation on the density field derived by Dean for Langevin particles [10]. This is important since Dean's derivation contains several subtleties. In particular, some have raised concerns about a possible hidden coarse-graining procedure that would explain why the ideal gas entropy appears, quite unexpectedly, in the Langevin equation. We end the paper with various technical comments, and possible applications and extensions of our formalism.

Let us start from the simplest situation - two sites labeled 1 and 2 between which particles hop back and forth with a Poisson rate $W_{12}$ and $W_{21}$. The (integer) number of particles on the two sites are $n_{1}$ and $n_{2}$. The variation of $n_{i}$ between $t$ and $t+\mathrm{d} t$ will be noted $\mathrm{d} J_{i}$ as is standard for Poisson jump processes [11]; it is not a small quantity since it is equal to 0 or \pm 1 , but the probability for it to be non zero is of order $\mathrm{d} t$. Of course, $\mathrm{d} J_{1}$ and $\mathrm{d} J_{2}$ are strongly correlated since a particle leaving site 1 lands on site 2 and vice-versa. More precisely, $\mathrm{d} J_{1}=-\mathrm{d} J_{2}=+1$ with probability $n_{2} W_{21} \mathrm{~d} t, \mathrm{~d} J_{1}=-\mathrm{d} J_{2}=-1$ with probability $n_{1} W_{12} \mathrm{~d} t$, and $\mathrm{d} J_{1}=\mathrm{d} J_{2}=0$ otherwise. We now introduce, à la MSRDJ, the generating function for the histories of the system: $Z(\{n, \hat{n}\})=\left\langle\prod_{t} \exp \left[\hat{n}_{1}(t)\left(\mathrm{d} J_{1}-\right.\right.\right.$ $\left.\left.\left.\mathrm{d} n_{1}\right)+\hat{n}_{2}(t)\left(\mathrm{d} J_{2}-\mathrm{d} n_{2}\right)\right]\right\rangle$, where the averaging $\langle\ldots\rangle$ is over the realizations of the Poisson jump processes. From 
the above rules, it is easy to find the result in the limit $\mathrm{d} t \rightarrow 0$ :

$$
Z(\{n, \hat{n}\})=\exp \int \mathrm{d} t\left[-\hat{n}_{1} \partial_{t} n_{1}-\hat{n}_{2} \partial_{t} n_{2}+n_{1} W_{12}\left(e^{\hat{n}_{2}-\hat{n}_{1}}-1\right)+n_{2} W_{21}\left(e^{\hat{n}_{1}-\hat{n}_{2}}-1\right)\right]
$$

Obviously, one could add different processes, such as for example on-site annihilation, where $\mathrm{d} J_{i}=-1$ with probability $n_{i} \mu \mathrm{d} t$, branching, where $\mathrm{d} J_{i}=+1$ with probabil- ity $n_{i} \nu \mathrm{d} t$, two-body annihilation where $\mathrm{d} J_{i}=-2$ with probability $n_{i}\left(n_{i}-1\right) \lambda \mathrm{d} t$, etc. With $N$ sites on a lattice, the total MSRDJ 'action' reads $S=\ln Z$ :

$$
S(\{n, \hat{n}\})=\int \mathrm{d} t\left[-\sum_{i} \hat{n}_{i} \partial_{t} n_{i}+\sum_{\langle i j\rangle} n_{i} W_{i j}\left(e^{\hat{n}_{j}-\hat{n}_{i}}-1\right)+\sum_{i} n_{i}\left\{\mu\left(e^{-\hat{n}_{i}}-1\right)+\nu\left(e^{\hat{n}_{i}}-1\right)+\lambda\left(n_{i}-1\right)\left(e^{-2 \hat{n}_{i}}-1\right)\right\}\right],
$$

where $\langle i j\rangle$ means that the sum is over all couples $i, j$. In addition, a factorized initial condition with distribution $p\left(n_{i}(0)\right)$ can be included by adding a contribution $\sum_{i}\left[-\hat{n}_{i}(0) n_{i}(0)+\ln g\left(\hat{n}_{i}(0)\right)\right]$, with $g(x)=\sum_{q} p(q) e^{q x}$. As it is customary in procedures à la MSRDJ [3] the average value over the stochastic dynamics of any observable $O$, a generic function of $\left\{n_{i}(t)\right\}$, equals the average of $O$ over the field theory characterized by the action (2) and the fields $n_{i}, \hat{n}_{i}$ which should be treated as continuous. This ends the derivation of the field theory that, as anticipated, turns out to be much more straightforward than the DP one.
We now want to consider the continuum limit of (21). In this aim we write $W_{i j}=W_{0} \exp \left(\left[U_{i}-U_{j}\right] / 2 T\right)$ where $U_{i}$ is an on-site potential (possibly time dependent), which varies on scales much larger than the lattice spacing $a$ and $T$ is the temperature. Restricting to nearest-neighbour hopping, we therefore write: $W_{i j}=W_{0}\left(1-a \mathbf{e}_{i j} \cdot \nabla U(\vec{x}, t) / 2 T\right)$, where $\vec{x}$ is the position in space of site $i$ and $\mathbf{e}_{i j}$ is the unit vector pointing from $i$ to $j$. Defining the local density field $\rho(\vec{x}, t)=n_{i} / a^{d}$ and $\hat{\rho}(\vec{x}, t)=\hat{n}_{i}$ and expanding to second-order in gradients, we finally obtain (in the $a \rightarrow 0$ limit, and with $\lambda=0$ for the time being - see below):

$$
\begin{aligned}
S(\{\rho, \hat{\rho}\}) & =\int \mathrm{d} t \mathrm{~d} \vec{x}\left[-\hat{\rho}(\vec{x}, t) \partial_{t} \rho(\vec{x}, t)+\rho(\vec{x}, t)\left(\mu\left(e^{-\hat{\rho}}-1\right)+\nu\left(e^{\hat{\rho}}-1\right)\right)\right] \\
& -\gamma \int \mathrm{d} t \mathrm{~d} \vec{x} \nabla \hat{\rho}(\vec{x}, t) \cdot \rho(\vec{x}, t) \nabla U(\vec{x}, t)+\gamma T \int \mathrm{d} t \mathrm{~d} \vec{x}\left[-\nabla \rho(\vec{x}, t) \cdot \nabla \hat{\rho}(\vec{x}, t)+\rho(\vec{x}, t)(\nabla \hat{\rho}(\vec{x}, t))^{2}\right],
\end{aligned}
$$

where $\gamma \equiv W_{0} a^{2} / 2 T$ is the mobility of the particles. Note that the above derivation is easily generalized to many other processes and is independent of the particular form of $W_{i j}$ as long as detailed balance is verified (although one could also consider non-potential force fields as well).

Before mapping this action onto the more standard DP form, we want to specialize to the case where $\mu=\nu=0$, and $U(\vec{x}, t)$ comes from a two-body interaction between the particles, i.e: $U(\vec{x}, t)=\int \mathrm{d} \vec{y} \rho(\vec{y}, t) V(\vec{x}-\vec{y})$. In this case, one finds that the above action is identical to the one obtained by using the standard MSRDJ representation for the following Langevin equation, derived by Dean for interacting Brownian particles:

$$
\partial_{t} \rho(\vec{x}, t)=\nabla \cdot\left[\gamma \rho(\vec{x}, t) \nabla \frac{\partial F[\rho]}{\partial \rho}+\sqrt{\rho(\vec{x}, t)} \vec{\eta}(\vec{x}, t)\right],
$$

where $\vec{\eta}$ is a Gaussian white noise with correlations $\left\langle\eta_{\alpha}(\vec{x}, t) \eta_{\beta}\left(\vec{x}^{\prime}, t^{\prime}\right)\right\rangle=2 \gamma T \delta_{\alpha \beta} \delta\left(\vec{x}-\vec{x}^{\prime}\right) \delta\left(t-t^{\prime}\right)$ and $F[\rho]$ is the effective free-energy which has the naively expected shape $F[\rho]=T \int \mathrm{d} \vec{x} \rho(\vec{x}) \ln \rho(\vec{x})+1 / 2 \int \mathrm{d} \vec{x} \mathrm{~d} \vec{y} \rho(\vec{x}) V(\vec{x}-$ $\vec{y}) \rho(\vec{y})$ [Note that Dean set the mobility $\gamma$ of the particles to unity]. This is quite remarkable, since $\rho(\vec{x}, t)$ in Dean's equation is the exact continuum microscopic density of the system, $\rho(\vec{x}, t)=\sum_{i} \delta\left(\vec{x}-\vec{r}_{i}(t)\right)$, before any coarse- 
graining $\left[r_{i}(t)\right.$ denotes the position of particle $i$ at time $t]$; it is therefore not at all trivial that the effective freeenergy should have a mean-field form. The fact that our derivation, which starts on a lattice and never uses Ito calculus, leads to the same action confirms the validity of Dean's analysis.

Let us now consider the following Cole-Hopf change of variables, from the above fields $\rho, \hat{\rho}$ to new fields $\phi, \hat{\phi}$ defined as:

$$
\rho(\vec{x}, t)=\hat{\phi}(\vec{x}, t) \phi\left(\vec{x}, t^{-}\right) ; \quad \hat{\rho}(\vec{x}, t)=\ln (\hat{\phi}(\vec{x}, t)) .
$$

The Jacobian of this transformation is 1 and thus the measure is preserved. The reason for $t^{-}=t-\epsilon$ will be discussed in the following. Note that this change of variable could also be defined on the discrete lattice and it was already considered in the literature [3, 21, 22, 23], but the precise connection that we establish here seems not to have been noted before. It is straightforward to show that the above action $S(\{\rho, \hat{\rho}\})$ transforms into:

$$
\begin{aligned}
S(\{\phi, \hat{\phi}\})= & \int \mathrm{d} t \mathrm{~d} \vec{x}\left\{\hat{\phi}\left[-\partial_{t} \phi+\gamma T \nabla^{2} \phi+\gamma \nabla(\nabla U \phi)\right]\right. \\
& +(\nu-\mu \hat{\phi}) \phi(1-\hat{\phi})\} .
\end{aligned}
$$

Setting $U=0$ in the above equation, we recover $e x$ actly the DP action for the problem of diffusing, branching and annihilating particle, usually derived in a rather thorny way from a second-quantization representation of the Master equation (up to some boundary terms, which for clarity will be discussed later). Note that for $\mu=\nu=0$, the above action can be seen as the two-field representation of the propagator of the non-Hermitian Fokker-Planck operator for particles diffusing in a potential field [14]. In addition, in the case of two-body interaction between the particles, the $\nabla[\nabla U \phi]$ term becomes $\nabla\left[\int \mathrm{d} \vec{y} \phi(\vec{y}, t) \hat{\phi}(\vec{y}, t) \nabla V(\vec{y}-\vec{x}) \phi(\vec{x}, t)\right]$. Let us finally remark that the mapping from the action (2) to its DP counterpart works also on the lattice, the continuum limit does not play any important role from this perspective.

The above action looks very close to a MSRDJ representation of the naive Langevin equation describing the problem if one interprets $\phi$ as a density and shifts $\hat{\phi} \rightarrow \hat{\phi}+1$ in order to have $\langle\hat{\phi}\rangle=0$ as in usual MSRDJ (see e.g. [3]). However this interpretation is problematic since, as mentioned in the introduction, the noise term is unphysical [7]. For example, in the case of diffusing particles with pair-wise interaction $(\mu=\nu=0)$ the "noise" term has a correlator $\left\langle\eta(\vec{x}, t) \eta\left(\vec{x}^{\prime}, t^{\prime}\right)\right\rangle=$ $2 \nabla_{x}\left[\phi(\vec{x})\left(\nabla_{x} V\right)\left(\vec{x}-\vec{x}^{\prime}\right) \phi\left(\vec{x}^{\prime}\right)\right]$. For a uniform density $\phi$ and $V(x)=V_{0} \exp \left(-x^{2}\right)$, the variance of the noise is a negative definite operator meaning that the noise has an imaginary part! On the other hand, we clearly see that this difficulty disappears when one uses the fields $\rho, \hat{\rho}$, which encode a very well defined underlying Langevin equation, albeit with non Gaussian, Poisson jump terms corresponding to particle creation and annihilation.

Let us now focus on some technical but important subtleties of the transformation (5). Consider pairannihilation $(A+A \rightarrow 0)$ with rate $\lambda$ on a single site. The corresponding contribution in the MSRDJ and DP actions are $\lambda \rho(\rho-1)\left(e^{-2 \hat{\rho}}-1\right)$ and $\lambda \phi^{2}\left(1-\hat{\phi}^{2}\right)$, which do not transform exactly into one another under (5). The underlying reason is that the two field theories correspond to different time discretizations. This makes a difference when the action contains non linear terms evaluated at the same position in time and space, because the response function $\left\langle\phi(\vec{x}, t) \hat{\phi}\left(\vec{x}, t^{\prime}\right)\right\rangle$ is discontinuous when $t=t^{\prime}$. This can be traced back to the fact that the fields $\phi$ and $\hat{\phi}$ are the coherent state representations of creation/annihilation operators of the theory, $a$ and $a^{+}$, which satisfy $\left[a, a^{+}\right]=1$. In the DP formalism the action is obtained after normal ordering [3] and as a consequence, $\hat{\phi}(\vec{x}, t) \phi(\vec{x}, t) \hat{\phi}(\vec{x}, t) \phi(\vec{x}, t)$ is in fact the continuous time limit of $\hat{\phi}(\vec{x}, t) \hat{\phi}(\vec{x}, t-\epsilon) \phi(\vec{x}, t-2 \epsilon) \phi(\vec{x}, t-3 \epsilon)$. Instead, the field theory obtained from (2) through the transformation (5) has no normal ordering and therefore the term $\lambda \rho^{2}(\vec{x}, t)$ is the continuous time limit of $\lambda \hat{\phi}(\vec{x}, t) \phi(\vec{x}, t-\epsilon) \hat{\phi}(\vec{x}, t-2 \epsilon) \phi(\vec{x}, t-3 \epsilon)$. Thus, to transform our field theory into DP, one has to use the transformation (5) and in addition to take care of the time discretization which amounts to do normal ordering, and to recover the -1 missing in the above example. An alternative way to make the exact connection between the two field theory is through the operator formalism. After having expressed the Master equation in terms of the operators $a, a^{+}$one does a canonical transformation $a=e^{-\rho^{+}} \rho, a^{+}=e^{\rho^{+}}$such that $\left[\rho, \rho^{+}\right]=1$. The operators $\rho, \rho^{+}$, originally introduced in [9], lead to a different representation of the Master equation. This then leads exactly, using again a coherent state representation, to the field theory that we derived above directly from underlying the stochastic process, including the correct boundary terms.

Another case where our strategy applies is interacting particles evolving under Newtonian dynamics, for which Doi also derived a field theory 15. Our previous starting point consisted in deriving exact stochastic equations for the density field and then applying the MSRDJ procedure to get the field theory. For Hamiltonian dynamics, one can derive an exact deterministic equation on the density in position $\vec{r}$ and momentum $\vec{p}$ space, $\rho(\vec{x}, \vec{p} ; t)=\sum_{i} \delta\left(\vec{r}-\vec{r}_{i}\right) \delta\left(\vec{p}-\vec{p}_{i}\right):$

$$
\begin{gathered}
\partial_{t} \rho(\vec{x}, \vec{p} ; t)=-\frac{1}{m} \vec{p} \cdot \partial_{\vec{x}} \rho(\vec{x}, \vec{p} ; t) \\
+\int d \vec{x}^{\prime} d \vec{p}^{\prime} \rho\left(\vec{x}^{\prime}, \vec{p}^{\prime} ; t\right) \partial_{\vec{x}} V\left(\vec{x}-\vec{x}^{\prime}\right) \cdot \partial_{\vec{p}} \rho(\vec{x}, \vec{p} ; t) .
\end{gathered}
$$

In this case only the initial conditions are stochastic. The MSRDJ field theory corresponding to this equation 
maps exactly onto Doi's using the transformation, akin to (5), $\phi(\vec{x}, \vec{p} ; t)=e^{-\hat{\rho}(\vec{x}, \vec{p} ; t)} \rho(\vec{x}, \vec{p} ; t)$ and $\hat{\phi}(\vec{x}, \vec{p} ; t)=$ $e^{\hat{\rho}(\vec{x}, \vec{p} ; t)}$ (note that $\hat{\rho}(\vec{x}, \vec{p} ; t)$ is the MSRDJ field conjugate to $\rho(\vec{x}, \vec{p} ; t))$.

Finally, our formalism in the continuum limit bridges the gap between the purely microscopic Fokker-Planck evolution operator and the hydrodynamics description studied recently in the mathematical physics literature. A method to handle both the hydrodynamic limit and large and rare fluctuations around it, has been developed in [8]. The starting point of this work is very close to the generating functional we used. However, in order to focus on hydrodynamics length-scales and timescales, as done in [8], one has to consider a conjugated field $\hat{n}_{i}=\hat{\rho}(x / L)$ that is constrained to varying only on length-scales of the order of the linear system size $L$. In this case the "action" or functional (2) becomes a function of the hydrodynamic density field that represents the average density inside very large boxes, and can be related to the rate functional introduced and studied in [8]. On the other hand, the stochastic equations corresponding to our continuum limit are valid on a scale $\ell$ much larger than the lattice spacing $a$ but much smaller than the system size $L$. As a consequence these allow one to tackle, with field theoretical techniques, dynamic phase transitions where the physically relevant length-scales are much larger than $a$ but not necessarily much larger than the (diverging) correlation length $\xi$. The hydrodynamic limit of [8] instead corresponds to length-scales much larger than $\xi$.

In conclusion our procedure allows one to derive rather straightforwardly a field theory different from, but dual of, the Doi-Peliti formalism. This could be useful in cases where this by now standard framework does not work, see e.g. [13]. It certainly avoids, contrarily to DP, very cumbersome computations due to normal ordering in cases in which the rates are complicated functions of the local density. Another advantage of our approach is that it is, almost by construction, directly related to stochastic equations on the density field. The representation in terms of a stochastic equation, especially after having taken the continuum limit, is particularly appealing. It can be helpful for numerical investigations 16] since it might be more efficient to integrate numerically than to simulate the original lattice model. Furthermore, stochastic equations are very useful to encode and study universality classes as it has been understood in the case of critical slowing down [17] and non-equilibrium phenomena such as surface growth [18]. Some applications of the results of this letter are underway, for example the study of the condensation phase transition in the zero range process [19]. From a more general perspective our results make clear that exact stochastic equations can be always obtained, thereby avoiding phenomenological guesses which, especially for off-equilibrium cases, can be very tricky [20].

Acknowledgments - We thank I. Dornic, C. Godrèche, F. Van Wijland for helpful and interesting discussions. A substantial part of this work has been done at the Isaac Newton Institute for Mathematical Sciences during the program "Principles of the Dynamics of Non-Equilibrium Systems". AA and GB are partially supported by the European Community's Human Potential Program contracts HPRN-CT-2002-00307 (DYGLAGEMEM).

[1] G. Tarjus, D. Kivelson, Jamming and Rheology: Dynamics on Microscopic and Macroscopic Scales, A. Liu, and S. Nagel, Taylor and Francis (2001).

[2] H. Hinrichsen, Adv. Phys. 49 (2000) 815. M. R. Evans, R. A. Blythe, Physica A (2002) 313110.

[3] J. Cardy, Lectures of the Troisième Cycle de la Suisse Romande, 1999. U.C. Täuber, Lecture Notes for Luxembourg Summer School "Ageing and the Glass Transition", cond-mat/0511743

[4] B. Delamotte, L. Canet, Condensed Matter Phys. 8 (2005) 163.

[5] S. P. Das and G. F. Mazenko, Phys. Rev. A 34, 2265 (1986); A. Andreanov, G. Biroli, A. Lefèvre, cond-mat/0510669

[6] J. P. Bouchaud and M. E. Cates, Phys. Rev. E 47, R1455 (1993). J. P. Doherty, M. A. Moore, J. M. Kim, and A. J. Bray, Phys. Rev. Lett. 72, 2041 (1994).

[7] M. J. Howard, U.C. Taüber, J. Phys. A: Math. Gen. 30 (1997) 7721.

[8] L. Bertini, A. De Sole, D. Gabrielli, G. Jona-Lasinio, C. Landim, cond-mat/0506664 J. Stat. Phys. 107 (2002), 635; Phys. Rev. Lett. 87 (2001), 040601.

[9] P. C. Martin, E. D. Siggia and H. A. Rose, Phys. Rev. A 8, 423 (1973); C. De Dominicis, J. Phys. C 1247 (1976); H. K. Janssen, Z. Phys. 24113 (1976).

[10] D. S. Dean, J. Phys A 29, L613 (1996).

[11] see e.g. K. Sato, Lèvy Processes and Infinitely Divisible Distributions, Cambridge University Press (1999).

[12] F. Van Wijland, Phys. Rev. E 63, 022101(2001).

[13] H.-K. Janssen, F. van Wijland, O. Deloubrière, U. C. Täuber, Phys. Rev. E 70 (2004) 056114.

[14] J. M. Luck, Nucl. Phys. B 225, 169 (1983).

[15] M. Doi, J. Phys A 9, 1465 (1976).

[16] I. Dornic, H. Chaté, M. A. Muñoz, cond-mat/0404105

[17] P.C. Hohenberg and B.I. Halperin, Rev. Mod. Phys., 49 (1977) 435.

[18] M. Kardar, G. Parisi and Y.C. Zhang Phys. Rev. Lett. 56 (1986) 889.

[19] G. Biroli, C. Godrèche and A. Lefèvre, unpublished

[20] M.A. Muñoz, APCTP Bull. 5 (2002) 5, cond-mat/0210645

[21] P. Grassberger, Z. Phys. B 47, 365 (1982).

[22] H. K. Janssen, Z. Phys. B 42, 151 (1981).

[23] M. E. Brachet and E. Tirapegui, Phys. Lett. A 81, 211 (1981). 